Cuadernos de Lingüística Hispánica nº 23

ISSN 0121-053X • ISSN en línea 2346-1829

Enero-Junio 2014, pp. 13-28

\title{
El léxico etnobiológico en Lengua mapuche*
}

HERM INIA NAVARRO HARTM ANN**

nherminia@gmail.com

Recepción: 18 de julio de 2013

Aprobación: 17 de noviembre de 2013

${ }^{-}{ }^{-}$Artículo de investigación científica. Este trabajo hace parte del proyecto PICT radicado en la UN de La Pampa sobre lingǘstica descriptiva y tipológica de las lenguas indígenas de Argentina, con especial énfasis en la región patagónica: mapudungun/ ranquel, tehuelche meridional o acndk'o ?a’jen, teushen, g̈̈nüna iájech tehuelche septentrional), selknam, hausch, yagan.

** Investigadora y maestranda en Lingüística por la Facultad de Humanidades y Ciencias de la Educación de la Universidad Nacional de La Plata, Rca. Argentina. profesora en Letras de la misma Universidad.

Integra un equipo de investigación que desarrolla el proyecto PICT radicado en la UN de La Pampa sobre lingüística descriptiva y tipológica de las lenguas indígenas de Argentina, con especial énfasis en la región patagónica: mapudungun/ ranquel, tehuelche meridional o aonek'o ?a?jen, teushen, gününa iájech tehuelche septentrional), selknam, hausch, yagan 


\title{
Resumen
}

Este trabajo forma parte de una investigación mayor encuadrada en un proyecto PICT de la Universidad Nacional de La Pampa sobre lingǘstica descriptiva y tipológica delas lenguas indígenas de Argentina, con especial énfasis en la región patagónica. Vinculada al grupo de lingüistas platenses se halla la viday la obra de Bertha K oessler-Ilg, investigadora alemana que recolectó y estudió tradiciones mapuches desde su llegada a la Patagonia argentina, en 1920, hasta su muerte en 1962. En este artículo se ejemplifica la terminología de fitónimos y zoónimos en mapudungun rastreados en la obra de la folklorista alemana; trabajamos sobre morfología de los términos de flora y fauna, con especial referencia al área neuquina. Se concluyó que existe una significativa motivación en el vocabulario etnobiológico mapuche, ya que para nombrar los elementos del entorno natural, los hablantes emplean, entre otros, procedimientos de simbolismo sonoro.

Palabras clave: mapudungun, lexicogénesis, fitonimia, zoonimia, simbolismo sonoro.

\section{Ethnobiological lexicon in the mapuchelanguage}

\begin{abstract}
A bstract:
This work is part of a larger research project that is part of the PICT project of the National University of La Pampa. This study analyzes the descriptive and typological linguistics of the indigen ous languages of Argentina, with special emphasis on the Patagonian region. Linked to the group of linguists from La Plata is the life and work of Bertha Koessler-Ilg, a German researcher who collected and studied Mapuche traditions since her arrival to La PatagoniaArgentina, in 1920, until her death in 1962. This article exemplifies the terminology of phytonyms and zoonyms in Mapudungun traced in the work of the $\mathrm{G}$ erman folklorist. The morphology of the terms of floraand fauna, with special reference to the Neuquén area, will be examined. It was concluded that there is significant motivation in the Mapuche ethnobiological vocabulary, since in order to name the elements of the natural environment, the speakers use procedures of sound symbolism, among others.
\end{abstract}

Key words: Mapudungun, lexicogenesis, phytonymy, zoonymy, sound symbolism. 


\section{Lelexiqueethnobiologiqueen langue mapuche}

\section{Résumé}

Ce travail fait partie d'une recherche plus large, encadrée dans un projet PICT de l'Université Nacional de la Pampa, sur linguistique descriptive et typologique des langues indigènes de l'Argentine, avec un accentuation spéciale dans la région patagonique. La vie et l'œuvre de Bertha Koessler-Ilg se trouve liée au groupe de linguistes platens elle était une chercheuse allemande qui a collecté et étudié des traditions mapurhs dès son arrivée à la Patagonie argentine en 1920, jusqu'à sa mort en 1962. D ans cet article on exemplifie la terminologie de phitonymes et des zoonymes en mapudungun suivis dans l'œuvre de la folkloriste allemande. Nous travaillons sur la morphologie des ternes de flore et faune, faisant une spéciale référence à la zone neuquine. On a conclu qu'il existe une motivation significative dans le vocabulaire ethnobiologique mapurhe, car pour nommer les éléments de l'entourage naturel, les locuteurs de la langue utilisant, entre autres, des procédures de symbolisme sonore.

M ots clés: mapudungun lexicogenèse, phitonymie, zoonomie, symbolisme sonore

\section{0 lexico etnobiologico em lengua mapuche}

\section{Resumo}

O trabalho faz parte de uma pesquisa matricial de um projeto PICT daUniversidade Nacional daPampa sobre linguística descritivay tipológica das línguas indígenas daArgentina, com especial ênfases na região da Patagônia. Vinculada ao grupo das linguísticas platenæs encontra-se a vida e obra da Bertha Koessler-Ilg, pesquisadora alemã que coletou e estudou tradições mapurhs desde a sua chegada na Patagonia Argentina em 1920, ate sua morte em 1962. Neste artigo se exemplifica a terminologia de fitónimos e zonimos em mapudungun rastreados na obra da folclorista alemã; trabalhamos sobre morfologia dos termos de flora e fauna, com especial ênfase na área nexquina Concluiu-se que existe uma significativa motivação no vocabulário etno-biológico mapurhe, visto que para nomear os elementos do entorno natural, os falantes usam procedimentos de símbolo sonoro.

Palavras C have: mapudungun léxico-gênesis, fitonimia, zonimia, simbolismo sonoro. 


\section{Introducción}

Este trabajo tratará de demostrar que el léxico etnobiológico de la lengua mapuche, como ocurre con gran parte de las lenguas indígenas del mundo, es altamente motivado. Para ello, se partirá del principio de arbitrariedad del lenguaje enunciado por Ferdinand de Saussure, se considerarán luego los conceptos de Sidney Lamb acerca de arbitrariedad y mo tivación, posteriormente se reflexionará acerca de las nociones de Brent Berlin sobre el simbolismo sonoro del lenguaje y, finalmente, se analizarán casos de la lengua mapuche, mapudungun en los que las palabras están motivadas.

D esde las investigaciones pioneras de Sapir $(1911,1929)$, se han estudiado los fenómenos que establecen algún tipo de relación directa entre la forma sonora y el significado de la palabra. Sin embargo, Sapir (en Langrage, 1921) dice que las lenguas de los pueblos tradicionales no se destacan por la abundancia de palabras imitativas como onomatopeyas o interjecciones. Las lenguas de las culturas más antiguas de América del Norte apenas tienen palabras imitativas o directamente carecen de ellas. En cambio, en lenguas de las culturas europeas centrales como el inglés 0 el alemán, se emplean numerosas onomatopeyas. Tal aseveración de Sapir es, a mi criterio, pertinente en las primeras etapas de la antropología lingüística. Los desarrollos posteriores de esta disciplina y sus afines: etnografía del habla, etnolingüística, etnopoética, amplían los campos de investigación extendiéndolos a innumerables lenguas nativas, que confirman o refutan principios del lenguaje enunciados para las lenguas centrales. Estos planteamientos, muchas veces, resultan novedosos en el campo de la Lingüística general. Es más, los estudios formales del lenguaje universal se sustentan mientras un recóndito lingüista, estudioso de una "pequeña lengua" (en la concepción de Whorf), no demuestre lo contrario. 


\section{D eSaussure y la arbitrariedad del signo lingüístico}

En su CursodeLingüstica Geneal, el lingüista suizo definió varios elementos, muchos dicotómicos, para describir la ciencia que, sin proponérselo, estaba fundando. Son ellos lengua y habla, diacronía y sincronía, significado-significante dentro del signo lingüístico. Al referirse a las características de este último dirá que está compuesto por un significado y un significante, y que es arbitrario. Con estas palabras da cuenta de la arbitrariedad del signo:

El lazo que une el significado y el significante es arbitrario; o bien, puesto que entendemos por signo el total resultante de la asociación de un significante con un significado, podemos decir más simplemente: el signo lingüístico es arbitrario.

Así, la idea de surno está ligada por relación alguna interior con la secuencia de sonidos surr que le sirve de significante; podría estar representada tan perfectamente por cualquier otra secuencia de sonidos. Sirvan de prueba las diferencias entre las lenguas y la existencia misma de lenguas diferentes: el significado 'buey' tiene por significante bué a un lado de la frontera franco española y böf (boetf) al otro, y al otro lado de la frontera franco germana es dks(Odhs).

La palabra arbitrario necesita también una observación. No debe dar idea de que el significante depende de la libre elección del hablante [... ] queremos decir que es inmotivado es decir, arbitrario en relación al significado, con el cual no guarda en la realidad ningún lazo natural. (2012, pp.144-146).

Plantea el lingüista el caso de las onomatopeyas como objeción posible a este principio:

Se podría uno apoyar en las onomatopeyas para decir que la elección del significante no siempre es arbitraria. Pero las onomatopeyas nunca son elementos orgánicos en un sistema lingüístico. Su número es, por lo demás, mucho menor de lo que se cree. Palabras francesas como foet, 'látigo' 0 gas 'doblar de campanas', pueden impresionar a ciertos oídos como una sonoridad sugestiva; pero para ver que no tienen tal carácter desde su origen, basta recordar sus formas latinas (foetderiva de fagus 'haya' y gases dassiam;; la cualidad de sus sonidos actuales, o mejor dicho, la que se les atribuye, es un resultado fortuito de la evolución fonética.

En cuanto a las onomatopeyas auténticas (las del tipo gugu, ticac, etc.) no solamente son escasas sino que su elección ya es arbitraria en cierta medida 
porque ya son la imitación aproximada y convencional de ciertos ruidos (francés cauaa, alemán uaumau, español gra gra). (2012, p. 146).

En el capítulo VI de su Curso, D e Saussure expondrá las diferencias entre lo arbitrario absoluto y lo arbitrario relativo, admitiendo que el principio fundamental de la arbitrariedad tiene grados, o bien, es un continuum entre lo radicalmente inmotivado y lo relativamente motivado, dos extremos entre los que oscilan todas las posibilidades de las lenguas del mundo. "El signo puede ser relativamente motivado" (2012, p. 240), dirá y ejemplificará con casos de composición y derivación, es decir, con procedimientos gramaticales: los numerales (dieinueve), ciertos sustantivos derivados (vaquero), los procedimientos de sufijación (limonero). D estaca la importancia que reviste para los lingüistas el hecho de admitir la limitación de lo arbitrario. "No hay lengua alguna en que no haya cosa motivada" (2012, p. 242). Entre los dos extremos, el mínimo de organización y el mínimo de arbitrariedad, se encuentran todas las variedades posibles de todas las lenguas del mundo. Allí estriba para Saussure la gran clasificación de las lenguas del mundo: aquellas en que lo inmotivado llega a su máximo son más lexicológicas, y aquellas en que se reduce al mínimo son más gramaticales (2012, pp. 242-243).

Hay concepciones contrarias al principio de la arbitrariedad que se centran en su opuesto, es decir, plantean el principio del lenguaje natural denominado "de la iconicidad". Lucía G olluscio (2005) lo define como la premisa por la cual las relaciones semánticas se reflejan en patrones formales (p. 255). El principio pragmático-cognitivo considerado se manifiesta mediante diversos recursos lingüísticos cognitivos, que mediante procesos de gramaticalización quedan fijados en la lengua como estrategias discursivas concretas.

En esta dirección van los desarrollos de la lingüística neurocognitiva de Sydney Lamb, quien realizó investigaciones sobre las lenguas indias de Norteamérica, específicamente las localizadas en el área en que se encuentra, California. La lengua estudiada por Lamb es el monachi.

\section{Sydney Lamb y los atisbos de motivación}

Por su parte, el lingüista neurocognitivo Sydney Lamb (2011) establece en su libro Senderos de cerebro un cierto cuestionamiento al principio de arbitrariedad propuesto por De Saussure. Se refiere a la contrapartida de lo arbitrario con ejemplos de lenguaje aparentemente motivado. Así analiza la "arbitrariedad": 
A rbitrariedad. La relación entre sonido y significado es en principio arbitraria. Si nos preguntamos “ ¿Por qué la rosa se llama rosa?” , la respuesta "porque se ve como una rosa" resulta nada satisfactoria. No parece haber conexión natural alguna entre el sonido y el significado. Este hecho puede verse a las claras en el caso de caballo cuyo significado se representa mediante sonidos bien diferentes en el inglés (harse), el francés (dheal), el alemán (Pferd), y el ruso (loshad).

Por otro lado, esta relación no es ni siempre ni necesariamente arbitraria. Por ejemplo, las vocales anteriores altas (articuladas con la boca apenas abierta), tienden a correlacionarse con objetos pequeños o conceptos de pequeñez. Así, un pdín es más pequeño que un pao y una pizca es más pequeña que una parión Pero existen muchas excepciones, como tal vez gंgantey corta Los lingüistas emplean el término icnicidadpara referirse a la no-arbitrariedad. En las estructuras de las oraciones y de unidades mayores abunda la iconicidad, en especial en lo que respecta al paso del tiempo. En la famosa sentencia de Julio César vini, vidi, vid "Vine, vi y conquisté", la secuencia de eventos se corresponde con la de las palabras. Existe otra forma de iconicidad, más abstracta y más susceptible de pasarse por alto. En la mayoría de las lenguas - con la notable excepción del inglés, cuyos lexemas provienen de muchas fuentes extranjeras- las formas fonológicas de los radicales nominales y verbales (y a menudo de otras categorías) coinciden mayormente en su estructura general. Tales formas reciben el nombre de FORMAS CANÓNICAS. Por ejemplo, la forma canónica de los radicales verbales y/ o nominales en algunas lenguas es CVCV o CVCCV (donde $\mathrm{C}$ significa consonante y $\mathrm{V}$, vocal). Además, los prefijos y los sufijos suelen ser más cortos que los radicales (la forma más común en las lenguas típicas es CV), cuasi-morfemas. Si bien suele definirse al morfema como la unidad mínima dotada de significado, a menudo encontramos partes de morfemas que parecen ostentar significado propio. Por ejemplo, en referencia al inglés, Bloomfield (1933) advirtió que la secuencia d- en side, surp sush sip etc., significa algo así como "levemente mojado . Como posible contrapartida en el español, podría sugerirse que la secuencia $\propto$ - (/ $\mathrm{kr}-/$ ) en aüientey cocante tiene incorporada el significado de "duro y quebradizo ; y que la secuencia - da (/ eta/ ) en palda, raquday palmda, evoca el significado "utilizado para golpear, si bien estos ejemplos no han sido documentados (p. 73)

D os conceptos destacados en el párrafo anterior para reforzar la iconicidad del lenguaje, vinculada a sus aspectos fonológicos: las vocales anteriores altas denotan tamaño pequeño. Existen morfemas que expresan por sí solos significado. 
En consonancia con estos planteos de Lamb se encuentran los desarrollos de Berlin.

\section{Brent Berlin y el simbolismo sonoro}

O tra vuelta de tuerca en la discusión entre motivación o arbitrariedad del lenguaje se torna evidente al analizar ejemplos del léxico etnobiológico de lenguas indígenas. Estosidiomas presentan un vocabulario etnobotánico y etnozoológico altamente motivado.

Para analizar el vocabulario etnobiológico del mapudungun nos basaremos en los conceptos de Brent Berlin (1992, 2006), quien desarrolla el punto de vista lingüístico de la etnobiología. Así, sugiere que, en el dominio etnobiológico, los nombres para la mayoría de los géneros nativos están gobernados por tres principios nomenclaturales: la descripción (literal o metafórica) de alguna propiedad observable del organismo, el simbolismo sonoro y la onomatopeya.

La propuesta de Berlin desafía la noción de la arbitrariedad del lenguaje, dado que una parte significativa de los vocabularios etnobotánicos y etnozoológicos está motivada en algún grado. El autor ha estudiado el tema de la asociación entre cualidad vocálica (ocurrencia de [i]) con el tamaño relativo de diferentes especies de aves en el huambisa (lengua jivaroana de Perú) y tres lenguas nativas americanas no emparentadas: waxampi (tupí), apalái (carib) y tzeltal (maya). Por lo que concluye que esa frecuencia es un patrón translingüístico en el léxico etnoornitológico.

Se conoce como simbolismo sonoro el fenómeno por el cual la forma sonora de un vocablo se relaciona directamente con el significado del mismo. Es un procedimiento fundado en el principio de iconicidad del lenguaje, que trasparenta la motivación de la lengua, no su arbitrariedad.

Es por esto que un autor como Hinton etal (1994) clasifica los fenómenos de simbolismo sonoro según los grados de proximidad y convencionalidad (motivación/ arbitrariedad) existente entre sonido y significado, diferenciando cuatro categorías: corporal, imitativo, sinestésico y convencional.

El primero corresponde a las interjecciones y sonidos involuntarios. El segundo comprende las palabras onomatopéyicas de sonidos del ambiente natural (golpes) y las representaciones de trinos y sonidos de otros animales. Sinestésico es el referido a la representación de propiedades visuales, táctiles o propioceptivas de los objetos (posición, orientación, movimiento, tamaño y forma). Es decir, 
que hay iconicidad entre alguna cualidad sensorial del significado o referente y la forma fonológica 0 algunas propiedades fonéticas de la palabra; tal es la correlación entre sonidos vocálicos y tamaño, o entre alargamiento vocálico y continuidad o duración de un evento. Como lo señalaba Lamb en el párrafo citado, existe una tendencia general por la que se usan vocales altas para expresar rasgos semánticos de tamaño pequeño o diminutivo. La cuarta categoría, denominada convencional, designa la asociación entre morfemas o conjuntos submorfémicos con determinados significados, propios de una determinada cultura; aseveraciones coincidentes con los planteos de Lamb.

Las tres primeras categorías presentan características translingüísticas similares, en tanto que en la última, la elección del segmento sonoro depende del vocabulario etnobiológico de cada lengua.

Para casos de simbolismo sonoro imitativo, tanto de onomatopeyas como de palabras eco, nos centraremos en la relación de sonidos no lingüísticos adaptados al sistema fonológico de la lengua (Hinton \& al. 1994; O swalt 1994: 293).

\section{Lexicogénesis en mapuzungun ¿tensión entre arbitrariedad y motivación?}

En mapuzungun o lengua mapuche, la iconicidad se expresa mediante varios recursos lingǘsticos. Analizaremos en este trabajo el simbolismo sonoro en el léxico etnobiológico. Presentamos algunos ejemplos de simbolismo sonoro imitativo:

\section{Keli keli}

'cernícalo, halconcito americano'. Falco sparverius

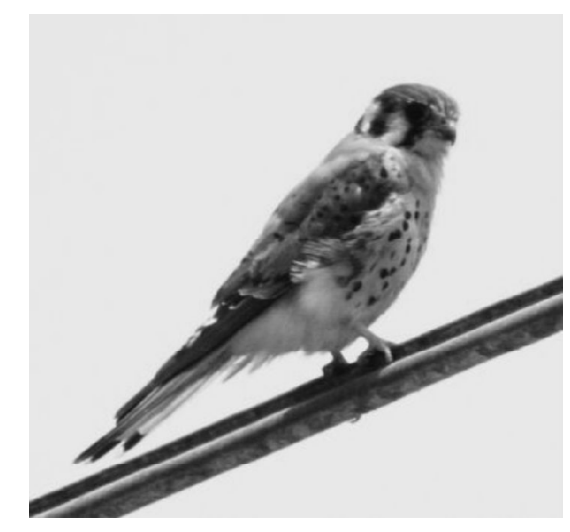




\section{Pitio}

'pitiwe, carpintero overo'. Cdaptes pitius

\section{Tue tue}

'tou tuo, lechucita pampa. Athene aniaularia

\section{Wiwi \\ ' koiwilla, coicoy, sapito chileno de cuatro ojos'. \\ Cystignathus bibronii}
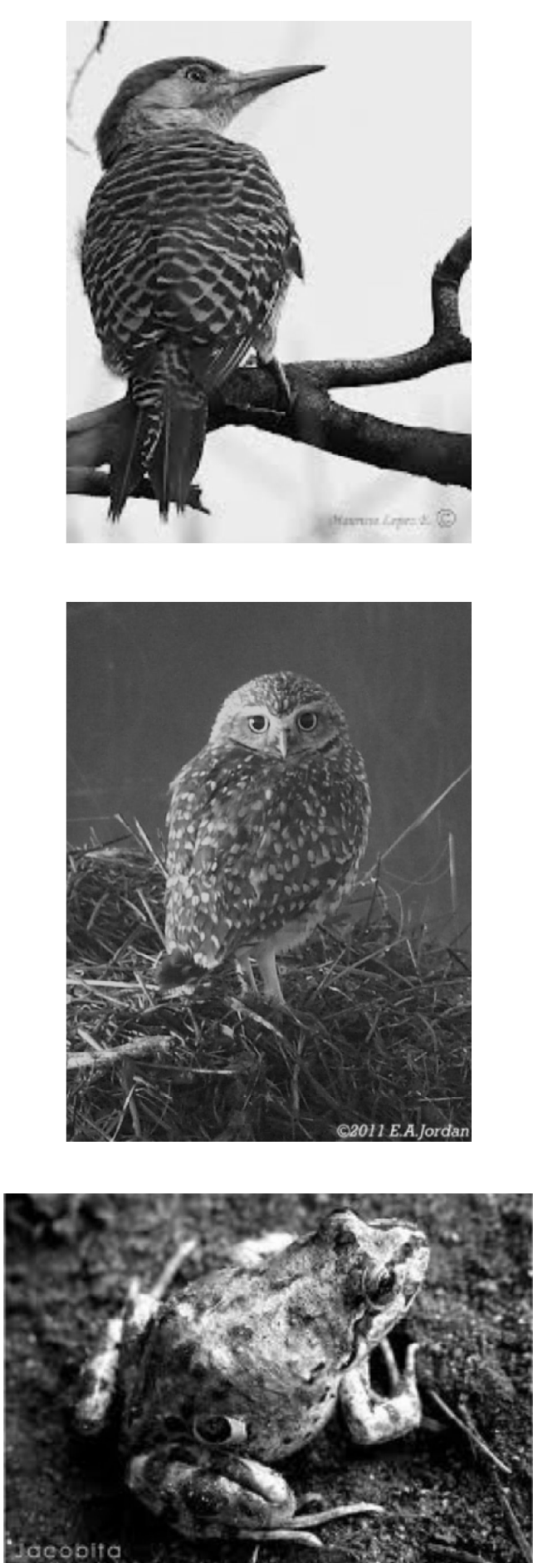
La mayoría son aves; la bibliografía consultada no da cuenta de la proporción de animales a los que corresponde un lexema/motivado en mapudungun. Pero sí existen datos para otras lenguas indígenas americanas, entre ellas, la lengua toba, familia guaycurú: "En nuestro corpus, cerca del $40 \%$ de los nombres tobas que designan aves están codificados mediante recursos de simbolismo sonoro" (Cúneo, 2011).

El simbolismo sonoro sinestésico es la simbolización acústica de fenómenos no acústicos (Hinton et al, 1994), es decir, que el material fonológico de una palabra es icónico de alguna cualidad del referente. Así se da la correlación entre sonido vocálico y tamaño, alargamiento vocálico y continuidad o duración de un evento o correspondencia entre sílaba y experiencia motora o sensorial. Palabras que denotan 0 connotan tamaño, familiaridad, cercanía, tienen vocales 0 consonantes con frecuencia acústica alta.

Hay tendencia en la asociación entre cualidad vocálicay frecuencia acústica y tamaño de la especie nombrada; se trata de un patrón translingüístico de simbolismo sonoro en el léxico etnozoológico. En mapudungun se manifiesta una frecuencia alta de las vocales [i], [u] en nombres de insectos, moluscos, mariscos y pájaros (en este último caso, el simbolismo sonoro no se apoya en la onomatopeya): es un patrón de simbolismo sonoro aso ciado al tamaño, propuesto por Berlin.

\section{Wilki}

'zorzal patagónico'

Turdus faldklandi

\section{Siwü}

'Jilguero'

Chrysanitnis banbata

\section{D iulliñ}

'moscardón, abejón'

\section{Pilui}

'babosa' (un molusco)

\section{R urü}

'zancudo' (un insecto) 


\section{Kufüll}

Toda clase de mariscos

En los casos precedentes, la motivación se apoya en los fonemas vocálicos "i", " $u$ ", dado que se comprueba la correlación existente entre la frecuencia de vocales altas y el tamaño del objeto designado. Pájaros e insectos, animales pequeños, cuyos nombres mapuches presentan numerosa concurrencia de las vocales altas " $i$ ", " $u$ "; las que se asocian, según Berlin y O hala, a objetos y seres de pequeño tamaño, o cercanos. Los ejemplificados son una muestra mínima de este patrón translingüístico en los zoónimos de pequeños animales en mapudungun. Estos nombres son bisílabos, con estructura CVCV. Las vocales altas se repiten, como en ejemplos 5 y 9, o alternan entre sí, con diferente orden, como en los ejemplos restantes.

En relación con los nombres de ranas y sapos, Berlin (1992, p. 250) observa que los sonidos ' $r$ ' (que incluyen: las vibrantes alveolares múltiples o till [r], simples o flap[ $\square$ ] y retroflejas [ $\square$ ], y la lateral dental/ alveolar [l]) son utilizados generalmente en laformación de estos nombres - en un corpus de cerca de treinta lenguas-. Por su parte, Hays (1994) testea este 'principio' en más de doscientas lenguas de Nueva G uinea y comprueba que los nombres de sapos y ranas utilizan desproporcionadamente los segmentos ' $g$ ' (que incluyen: las velares oclusivas $[\mathrm{k}]$ y $[\mathrm{g}]$, fricativas $[\mathrm{x}]$ y $[\square]$, y la oclusión glotal $[\square]$ ) en estas lenguas. Concluye que tanto los sonidos ' $\mathrm{g}$ ' como ' $r$ ' se usan significativamente en nombres genéricos de ranas y sapos. Estos nombres, por lo tanto, no parecen ser lexemas arbitrarios. La motivación fonológica se proyecta al nivel léxico.

En mapuzungun, los nombres de batracios corroboran este principio; sapos, ranas y escuerzos en mapuche se denominan:

\section{Lafatra}

'sapo'.

\section{Trawültrawül}

'Sapo', en dialecto en nguluche (mapuche occidental o chileno)

\section{A rümko}

(lit: arümko o ngenkka guardador del agua)

'sapo verde, rayado' 


\section{Koiwilla}

'coicoy, sapo pequeño cuya voz suena wiwi o coicoi'

Cystignatuhus bibronii

\section{Küran wakaq}

'sapo monstruo'

\section{Llüngki}

'rana',

\section{Pakar, pakara, pakur \\ 'escuerzo' \\ Bufo spinatosus}

Los ejemplos 11 y 12 presentan la vibrante alveolar retrofleja, muy habitual en mapudungun.

Mientras en 13, 14, 15, 16 y 17 observamos las velares oclusivas $\mathrm{k}$ y g, con presencia de vibrante alveolar simple en 13,15 y 17 . A sí corroboramos que en mapudungun los fonemas " $r$ " $\mathrm{y}$ " $g$ " se usan con gran frecuencia en nombres de batracios, como en gran parte de las lenguas americanas estudiadas por Berlin.

Encontramos numerosos zoónimos en los textos de Tradidiones Araucanas TomoI, de la folklorista alemana-argentina Bertha Koessler-Ilg. Por ejemplo, la explicación en torno al ave llamado cernícalo, cuyo nombre es kellengkelleng(p. 35). En nota de la autora se enuncia que el ave según la creencia, acompaña a los viajeros, adelantándose para anunciar su llegada. A él se refieren dos canciones de las recogidas por Augusta en sus Leeturas Arauramas (p. 324, canción nº 13; y p. 331, canción $\mathrm{n}^{\circ}$. 32).

Es significativa la semejanza entre los nombres de algunas aves, de rapiña o no. Augusta, en su Diccionario, interpreta que el cernícalo ha sido llamado kdikdi por onomatopeya de su grito. Sin embargo, en la misma página, registra el nombre del pájaro llamado "cola de palo" (kdekkden) interpretándolo como repetición de la palabra kden (cola). Todavía, en la misma página, aparece el cernícalo por el nombre kdengkdeng Por otra parte, bajo la voz "cernícalo", se lee llequleqken leqdeqken, kdike, kdikdi. En Moesbach (Vida y costumbres de los indígenas araucanos) se registran en páginas 11 y 112, dos de los nombres del 
cernícalo citados por Augusta, el nombre del "cola de palo", y el del chuncho (killkill). Todos estos nombres coinciden en su reduplicación y, aproximadamente, en su sílaba inicial. Esta reduplicación contribuye al carácter motivado de los signos. D el mismo tipo, es el nombre del peuco (kehiikehii). Lo más notable es que el kdengkdengo cernícalo difiere notablemente del kdenkdeno "cola de palo", siendo este último la única, de entre todas las aves mencionadas, que no es carnívora.

Los anotadores Albarracín Sarmiento y Estela de Souza completan: “En quechua, el cernícalo se llama killikilli, que además significa "listado, enflecado, ribeteado". También se llama killidhu, killimykillimaha

Respecto del tero (p. 174): BKI menciona la explicación dada por Vuletin y luego realiza su propio análisis: "Los diversos nombres araucanos del tero (posiblemente uno solo), lo mismo que los de otras varias aves, dirigen hacia el verbo kettüno kültrün

Qudtehue, qudtregil, qudtren, alteo, tegil, treguil, tréde, trüqudl. Para Vuletin son compuestos de dos sílabas:

a) -kel- (o - küll- 0 -kul- 0 -kl- 0 -guil- 0 - gül- 0 -gul- 0 -glo-)

b) -te- (o - tre- 0 - trü- 0 - ter-)

Puestas en uno u otro orden, una a continuación de otra.

La sílaba ke, etc. (y también kill- y keli-) en la formación de nombres de aves, por lo común como sílaba inicial reduplicada, parece vincularse con el verbo kedtrün: elevarse en el aire el pájaro o el sol, así como con los verbos de la misma familia: ketrüleno kütrulen estar suspendido en el aire; ketrüpun quedar suspendido en el aire lo que se ha tirado hacia arriba. Y en este caso, surge la duda en considerar los nombres como onomatopéyicos o como raíces nominales.

\section{C ondusiones}

Hay muchos ejemplos de motivación en el vocabulario etnobiológico mapuche, ya que para nombrar los elementos del entorno natural, los hablantes emplean procedimientos de simbolismo sonoro que tensionan la relación motivación/ arbitrariedad. Se pone de manifiesto tanto en los casos del simbolismo sonoro imitativo, como analizamos con las onomatopeyas, como con el sinestésico al considerar fragmentos de material fonológico que implican significado, tal sucede con el nombre de los batracios. 
Las onomatopeyas en mapuche son muy numerosas y originan un número significativo de vocablos que las incorporan, mayormente reduplicadas. Esta característica las diferencia de los casos analizados por De Saussure para las onomatopeyas de las lenguas europeas clásicas. Analizamos ejemplos de animales, especialmente aves, cuyo nombre refiere su canto.

El recurso de la reduplicación contribuye a la motivación, dado que es un subprincipio de la iconicidad del lenguaje. En el caso del mapudungun está ligado a varias funciones, no solo a la onomatopéyica, aunque es esta la principal en el vocabulario etnoornitológico. En los nombres de insectos, análisis posteriores permitirán extraer conclusiones.

Los nombres onomatopéyicos no son meramenteruidos, gritos involuntarios o palabras que se utilizan solo una vez, sino que constituyen ítemes léxicos convencionalizados, listados en los diccionarios y con un significado específico, es decir, se hallan lexicalizados.

Se comprueba el principio de iconicidad del lenguaje en la asociación entre frecuencia acústica, cualidad vocálica (fonemas [i], [u]) y tamaño de la especie y en la existencia de fonemas y morfemas con significado propio. A mbos son casos de simbolismo sinestésico, planteados por Lamb y Berlin.

\section{R eferencias bibliográficas}

Augusta, F. (1910). Leturas araucamas Santiago de Chile: Prefectura Apostólica.

Augusta, F.(1916). Dicionario mapuche españd-mapudhe Santiago de Chile: Imprenta Universitaria.

Berlin, B. (1984). Evidence for pervasive synesthetic sound symbolism in ethnozoological nomenclature. En: Leanne Hinton, Johanna Nichols y John O hala pp. 76-93).

Berlin, B. (1992). Ethndidojical ClassificationPrimiples of Cateegrizationof PlantsandAnimals in traditional soieties New Jersey: Princeton University Press.

Berlin, B. (2006). The First Congress of Ethnozoological Nomenclature.Ethnobiology and the Science of Human kind En: Jarnal of theRgal Anthropdogical Institute, vol. 12. p.p. 23-44. Matthew Engelke.

Coña, P. y Ernesto Wilhem de M. (1984). LanœoPasaal œ̃̃añitualpazugun=Tetimoniode 
un caaquemapurhe Santiago: Pehuén.

Cúneo, P. (2012). Clasificadón nominal y fomadón de palabras en tdba (familiagrayauú), con espeial referenia al léxico enddidógica Tesis de D octorado en Filosofía y Letras. UBA. FFyL, Universidad de Buenos Aires. Facultad de Filosofía y Letras.

De Saussure, F. (2012). CursodeLingüísica General. Buenos Aires: Losada. Recuperado de http:/ / www.misaves.com.ar/ fotos/ 380_D.jpg; http:/ / www.avesdelima.com/ fsierra/ ciene_cernicalo2.jpg; http:/ / www.google.com.ar/ imgres?imgurl=http:/ / www.jacobita.cl/ sitio/ wp-

Hinton, L., Johanna Nicholsy John O hala(eds.). (1994). Saund Symbdism Cambridge: Cambridge University Press.

KoesslerIlg, B. (1962).Tradidiones araucanas Tom I. Instituto de Filología. Facultad de Humanidades y Ciencias de la Educación. Universidad Nacional de La Plata.

Lamb, S. (2011). Senderos de cerdora Universidad de Mar del Plata: EUDEM.

Navarro, H. (2012). Bertha KoeslerIlg y las etimologías en mapuzungunI Jomadas de Histaria dela Lingüística Facultad de Filosofía y Letras. Buenos Aires: UBA.

O hala, J. (1997). Sound Symbolism.Proc 4th Seail International Conference on Lingristics [SICOL]: 98-103.

content/ uploads/ 2008/ 10/ sapito-4-ojos-01.jpg\&imgrefurl=http:/ / www.jacobita.cl/ fotografia/ el-sapito-de-cuatro-ojos $\& \mathrm{~h}=450 \& \mathrm{w}=600 \& \mathrm{sz}=113 \&$ tbnid $=5 \mathrm{BM7z}$ Cm06SgeeM:\&tbnh=93\&tbnw=124\&zoom=1\&usg= _xrU04H86T AQLJ2yXL vxgSYUThnY=\&docid=VzzSn2U2iQ honM\&sa=X \&ei=KgHO UaqoCoHwiwLBt ID 4Cg\&ved=0CCwQ 9Q E wAA \& dur=1301 